\title{
Stochastic Banach principle in operator algebras
}

\author{
by \\ Genady Ya. Grabarnik (Hawthorne, NY) \\ and LaUra Shwartz (Pretoria)
}

\begin{abstract}
The classical Banach principle is an essential tool for the investigation of ergodic properties of Cesàro subsequences. The aim of this work is to extend the Banach principle to the case of stochastic convergence in operator algebras. We start by establishing a sufficient condition for stochastic convergence (stochastic Banach principle). Then we prove stochastic convergence for bounded Besicovitch sequences, and as a consequence for uniform subsequences.
\end{abstract}

1. Introduction and preliminaries. In this paper we establish a stochastic Banach principle. The Banach principle, one of the most useful tools in "classical" pointwise ergodic theory, was used to give an alternative proof of the Birkhoff-Khinchin individual ergodic theorem. Typical applications of the principle are Sato's theorem for uniform subsequences [17] and the individual ergodic theorem for Besicovitch bounded sequences [16]. Noncommutative analogues for the (bilateral) almost everywhere convergence can be found in [9], [2].

In this paper we establish the Banach principle for convergence in measure (stochastic Banach principle) in Theorem 2.3, which is then reformulated in a form convenient for applications (Theorem 2.4). We apply it to give a simplified proof of the stochastic ergodic theorem (cf. [10]). We also establish stochastic convergence for Sato's uniform subsequences (Theorem 3.6) and a stochastic ergodic theorem for Besicovitch bounded sequences (Theorem 3.5$)$.

Note that the stochastic Banach principle is new even in the commutative case. Indeed, it is well known (see for example [13]) that there are Cesàro

2000 Mathematics Subject Classification: Primary 46L51; Secondary 37A30.

Key words and phrases: Banach principle, von Neumann algebras, non-commutative ergodic theorems, stochastic convergence.

The second author is grateful to her Mentor, Dr. Louis E. Labuschagne (UNISA, South Africa) for constant support and careful reading of the paper.

The authors are grateful to the referee for useful suggestions. 
averages constructed for automorphisms that converge in measure and do not converge almost everywhere. This implies that although the condition of Theorem 2.3 is satisfied in this case, the pointwise Banach principle condition is not.

Throughout the paper we denote by $M$ a von Neumann algebra with a semi-finite normal faithful trace $\tau$ acting on a Hilbert space $\mathfrak{H}$, and by $P(M)$ the set of all orthogonal projections in $M$.

Recall the following definitions (taken from the papers by Segal [18], Nelson [15], Yeadon [21], Fack and Kosaki [6]):

Definition 1.1. A densely defined closed operator $x$ affiliated with a von Neumann algebra $M$ is called $(\tau)$-measurable if for every $\varepsilon>0$ there exists a projection $e \in P(M)$ with $\tau(\mathbb{I}-e)<\varepsilon$ such that $e(\mathfrak{H}) \subset \mathfrak{D}(x)$, where $\mathfrak{D}(x)$ is the domain of $x$.

The space of all $(\tau)$-measurable operators affiliated with $M$ is denoted by $S(M)$ or $S(M, \tau)$.

For convenience, for a self-adjoint $x \in S(M)$ we denote by $\{x>t\}$ the spectral projection of $x$ corresponding to the interval $(t, \infty)$.

Definition 1.2. A sequence $\left\{x_{n}\right\}_{n=1}^{\infty}$ converges to 0 in measure if for every $\varepsilon, \delta>0$ there exist an integer $N_{0}$ and a set of projections $\left\{e_{n}\right\}_{n \geq N_{0}} \subset$ $P(M)$ such that $\left\|x_{n} e_{n}\right\|_{\infty}<\varepsilon$ and $\tau\left(\mathbb{I}-e_{n}\right)<\delta$ for $n \geq N_{0}$.

REMARK 1.1. We will use the terms converges in measure and converges stochastically interchangeably.

Definition 1.3. Let $x \in S(M)$ and $t>0$. The $t$ th singular number of $x$ is defined as

$$
\mu_{t}(x)=\inf \{\|x e\|: e \in P(M) \text { with } \tau(\mathbb{I}-e) \leq t\} .
$$

REMARK 1.2. Note that the measure topology is defined in Fack and Kosaki [6] as the linear topology with the fundamental system of zero neighborhoods given by $V(\varepsilon, \delta)=\{x \in S(M)$ : there exists $e(x, \varepsilon, \delta) \in P(M)$ with $\|x e\|<\varepsilon$ and $\tau(\mathbb{I}-e)<\delta\}$. By Nelson's [15] result, the set of all $\tau$ measurable operators is a complete topological *algebra. Since the measure topology has a countable fundamental system of zero neighborhoods, $S(M)$ is sequentially complete.

Definition 1.4. Denote by $\lambda_{t}(x)$ the distribution function of $x$ defined as

$$
\lambda_{t}(x)=\tau\left(E_{(t, \infty)}(|x|)\right), \quad t \geq 0,
$$

where $\left.E_{(t, \infty)}(|x|)\right)$ is the spectral projection of $x$ corresponding to the interval $(t, \infty)$. 
REMARK 1.3. For $x \in S(M)$, we have $\lambda_{t}(x)<\infty$ for large enough $t$ and $\lim _{t \rightarrow \infty} \lambda_{t}(x)=0$. Moreover, the map $\mathbb{R} \ni t \mapsto \lambda_{t}(x)$ is non-increasing and continuous from the right (since $\tau$ is normal and $\left\{|x|>t_{n}\right\} \uparrow\{|x|>t\}$, hence in the strong operator topology, as $\left.t_{n} \downarrow t\right)$. The distribution $\lambda_{t}(x)$ is a non-commutative analogue of the distribution function in classical analysis (see [6, p. 272], or [19]).

We need the following properties of $\mu_{t}(x)$ (see for example [21, Proposition 2.4], or [6, Lemma 2.5]):

Lemma 1.1. Let $x, y \in S(M)$.

(i) The map $\mathbb{R}_{+} \ni t \mapsto \mu_{t}(x)$ is non-increasing and continuous from the right. Moreover, $\lim _{t \downarrow 0} \mu_{t}(x)=\|x\|_{\infty} \in[0, \infty]$.

(ii) $\mu_{t}(x)=\mu_{t}(|x|)=\mu_{t}\left(x^{*}\right)$ and $\mu_{t}(\alpha x)=|\alpha| \mu_{t}(x)$ for $\alpha \in \mathbb{C}, t>0$.

(iii) $\mu_{t}(x) \leq \mu_{t}(y)$ for $0 \leq x \leq y, t>0$.

(iv) $\mu_{t+s}(x+y) \leq \mu_{t}(x)+\mu_{s}(y)$ for $t, s>0$.

(v) $\mu_{t}(y x z) \leq\|y\|_{\infty}\|z\|_{\infty} \mu_{t}(x)$ for $y, z \in M, t>0$.

(vi) $\mu_{t+s}(y x) \leq \mu_{t}(x) \mu_{s}(y)$ for $t, s>0$.

2. Stochastic Banach principle. We first formulate some conditions equivalent to stochastic convergence (cf. [6, Lemma 3.1]).

Lemma 2.1. Let $M, \tau$ be as before. Consider the following conditions:

(i) The sequence $\left\{x_{n}\right\}_{n=1}^{\infty}$ converges to 0 in measure.

(ii) For every $\varepsilon, \delta>0$ there exist $0<\delta^{\prime}<\delta$ and an integer $N_{0}$ such that for $n \geq N_{0}$,

$$
\mu_{\delta^{\prime}}\left(x_{n}\right)<\varepsilon .
$$

(iii) For every $\varepsilon, \delta>0$ and $p \in P(M)$ with $\tau(p)<\infty$ there exist an integer $N_{0}$ and a sequence $\left\{e_{n}^{\prime}\right\}_{n \geq N_{0}} \subset P(M)$ with $e_{n}^{\prime} \leq p$ such that

$$
\left\|x_{n} e_{n}^{\prime}\right\|_{\infty}<\varepsilon \quad \text { and } \quad \tau\left(p-e_{n}^{\prime}\right)<\delta \quad \text { for } n \geq N_{0} .
$$

Then (i) $\Leftrightarrow($ ii) $\Rightarrow$ (iii). If $\tau$ is finite then (iii) $\Rightarrow(\mathrm{i})$.

Proof. (ii) $\Rightarrow$ (i). The condition $\mu_{\delta^{\prime}}\left(x_{n}\right)<\varepsilon$ yields a sequence $\left\{e_{n}\right\}_{n=1}^{\infty}$ of projections with $\left\|x_{n} e_{n}\right\|<2 \varepsilon$ and $\tau\left(\mathbb{I}-e_{n}\right)<\delta^{\prime}$.

(i) $\Rightarrow$ (ii) follows from the definition of measure convergence 1.2.

(i)\&(ii) $\Rightarrow$ (iii) follows from the inequality $\tau(p-p \wedge q)=\tau(p \vee q-q) \leq$ $\tau(\mathbb{I}-q)$, hence the sequence $\left\{e_{n}^{\prime}=e_{n} \wedge p\right\}_{n=1}^{\infty}$ satisfies (iii) (here the projections $e_{n}$ are defined in the proof of (ii) $\Rightarrow(\mathrm{i})$ ).

The case when $\tau$ is finite follows immediately, since $\tau(\mathbb{I})<\infty$.

We need the following technical statement, which is interesting in its own right: 
LEMmA 2.2. Let $x, y \in S(M)$ be self-adjoint, and $t, s$ positive real. Then

$$
\lambda_{t+s}(x+y) \leq \lambda_{t}(x)+\lambda_{s}(y)
$$

Proof. Indeed,

$$
\begin{aligned}
\||(x+y)|(\mathbb{I}- & \{|x|>t\}) \wedge(\mathbb{I}-\{|y|>s\}) \| \\
= & \|(x+y)(\mathbb{I}-\{|x|>t\}) \wedge(\mathbb{I}-\{|y|>s\})\| \\
\leq & \|x(\mathbb{I}-\{|x|>t\}) \wedge(\mathbb{I}-\{|y|>s\})\| \\
& +\|y(\mathbb{I}-\{|x|>t\}) \wedge(\mathbb{I}-\{|y|>s\})\| \\
= & \||x|(\mathbb{I}-\{|x|>t\}) \wedge(\mathbb{I}-\{|y|>s\})\| \\
& +\||y|(\mathbb{I}-\{|x|>t\}) \wedge(\mathbb{I}-\{|y|>s\})\| \\
\leq & \||x|(\mathbb{I}-\{|x|>t\})\|+\||y|(\mathbb{I}-\{|y|>s\})\| \leq t+s .
\end{aligned}
$$

Here the first and second equalities follow from the equality $\left\||z| u_{z}^{*} u_{z}|z|\right\|=$ $\left\||z|^{2}\right\|=\left\|z^{*} z\right\|$, where $z \in M_{h}, u_{z}$ is a partial isometry from $M$ such that $z=u_{z}|z|$, and

$$
u_{z}^{*} u_{z}=l(z), \quad u_{z} u_{z}^{*}=r(z),
$$

where $l(z)$ (resp. $r(z)$ ) is the left (resp. right) support of $z$. Inequality (4) means that

$$
\mu_{\lambda_{t}(x)+\lambda_{s}(y)}(x+y) \leq t+s .
$$

Let $\xi$ be a vector from a Hilbert space $\mathfrak{H}$, and suppose that

$$
\xi \in\{|x+y|>s+t\} \mathfrak{H} \cap(\mathbb{I}-\{|x|>t\}) \wedge(\mathbb{I}-\{|y|>s\}) \mathfrak{H} .
$$

Then

$$
((t+s)\|\xi\|)^{2}<(|x+y| \xi,|x+y| \xi)=((x+y) \xi,(x+y) \xi) \leq((t+s)\|\xi\|)^{2} .
$$

Here the first inequality follows from the inclusion $\xi \in\{|x+y|>s+t\} \mathfrak{H}$, the equality follows from the spectral decomposition (5), and the second inequality follows from the inclusion $\xi \in(\mathbb{I}-\{|x|>t\}) \wedge(\mathbb{I}-\{|y|>s\}) \mathfrak{H}$.

Inequality (8) implies that $\|\xi\|=0$ or, in other words,

$$
\{|x+y|>s+t\} \wedge((\mathbb{I}-\{|x|>t\}) \wedge(\mathbb{I}-\{|y|>s\}))=0 .
$$

Hence,

$$
\begin{aligned}
\{|x+y| & >t+s\}=\{|x+y|>t+s\} \\
& -\{|x+y|>t+s\} \wedge((\mathbb{I}-\{|x|>t\}) \wedge(\mathbb{I}-\{|y|>s\})) \\
\sim & \{|x+y|>t+s\} \vee((\mathbb{I}-\{|x|>t\}) \wedge(\mathbb{I}-\{|y|>s\})) \\
& \quad-((\mathbb{I}-\{|x|>t\}) \wedge(\mathbb{I}-\{|y|>s\})) \\
\leq & \mathbb{I}-((\mathbb{I}-\{|x|>t\}) \wedge(\mathbb{I}-\{|y|>s\}))=\{|x|>t\} \vee\{|y|>s\} .
\end{aligned}
$$


Here $\sim$ denotes projection equivalence. Since the trace $\tau$ is invariant on equivalent projections,

$$
\begin{aligned}
\tau(\{|x+y|>t+s\}) & \leq \tau(\{|x|>t\} \vee\{|y|>s\}) \\
& \leq \tau(\{|x|>t\})+\tau(\{|y|>s\}) .
\end{aligned}
$$

Hence, inequality (3) is valid.

Theorem 2.3. Let $(B,\|\cdot\|)$ be a Banach space and $\Sigma=\left\{A_{n}: n \in \mathbb{N}\right\}$ a set of linear operators $A_{n}: B \rightarrow S(M)$.

(i) Suppose that there exists a function $C: \mathbb{R}_{+} \rightarrow \mathbb{R}_{+}$with $\lim _{\lambda \rightarrow \infty} C(\lambda)$ $=0$ and

$$
\sup _{n \in \mathbb{N}} \tau\left(\left\{\left|A_{n}(b)\right|>\lambda\|b\|\right\}\right) \leq C(\lambda)
$$

for every $b \in B$ and $\lambda \in \mathbb{R}_{+}$. Then the subset $\widetilde{B}$ of $B$ where $A_{n}(b)$ converges in measure is closed in $B$.

(ii) Conversely, if $A_{n}$ is a set of continuous-in-measure maps from $B$ into $S(M)$ and for all $b \in B$ and $\lambda \in \mathbb{R}_{+}$,

$$
\lim _{\lambda \rightarrow \infty} \sup _{n \in \mathbb{N}} \tau\left(\left\{\left|A_{n}(b)\right|>\lambda\right\}\right)=0,
$$

then there exists a function $C: \mathbb{R}_{+} \rightarrow \mathbb{R}_{+}$with $\lim _{\lambda \rightarrow \infty} C(\lambda)=0$ and

$$
\sup _{n \in \mathbb{N}} \tau\left(\left\{\left|A_{n}(b)\right|>\lambda\|b\|\right\}\right) \leq C(\lambda) .
$$

Part (i) means that under the condition of linear uniform boundedness (11), the set of stochastic convergence is closed.

Part (ii) means that if the set of uniform boundedness is closed, then linear uniform boundedness holds on this set.

Note that although the condition in (ii) looks more restrictive, it is similar in nature to that in (i), since everything could be restricted to the closure of the set where uniform boundedness holds (it is also a Banach space).

Proof. (i) We first show that condition (11) implies continuity of the operators in $\Sigma$. Let $\left\{b_{k}\right\}_{k=1}^{\infty}$ be a sequence in $B$ converging to $b \in B$. Then for $\lambda, \varepsilon \in \mathbb{R}_{+}$satisfying $2 \lambda \sup _{k \geq n}\left\|b-b_{k}\right\|<\varepsilon$, as $k \rightarrow \infty$ we have

$$
\begin{aligned}
\tau\left(\left\{\left|A_{n}\left(b_{k}\right)-A_{n}(b)\right|>\varepsilon\right\}\right) & \leq \tau\left(\left\{\left|A_{n}\left(b_{k}-b\right)\right|>\lambda\left\|b_{k}-b\right\|\right\}\right) \\
& \leq C\left(\lambda\left\|b_{k}-b\right\|^{-1}\right) \rightarrow 0 .
\end{aligned}
$$

Hence $A_{n}$ is continuous. Note that the inequality follows from the fact that the right-hand side of (11) is independent of the norm of $b$.

There exists a subsequence $b_{k_{j}}$ of $b_{k}$ such that the sequence $x_{j}=$ $\lim _{n \rightarrow \infty} A_{n}\left(b_{k_{j}}\right)$ converges stochastically (bear in mind that by Remark 1.2 
the algebra $S(M)$ is sequentially complete). In order to establish this statement, by (11) we find a sequence $\left\{k_{i}^{\prime}\right\}_{i=1}^{\infty}$ such that

$$
\begin{aligned}
\tau\left(\left\{\left|A_{n}\left(b_{k_{j}^{\prime}}-b_{k_{j+l}^{\prime}}\right)\right|>2^{-j}\right\}\right) \leq 2^{-j} & \text { for all } n, \\
\tau\left(\left\{\left|A_{n}\left(b_{k_{j}^{\prime}}-b\right)\right|>2^{-j}\right\}\right) \leq 2^{-j} & \text { for all } n .
\end{aligned}
$$

This can be done since $b_{n} \rightarrow b$ as $n \rightarrow \infty$, and $C(\lambda) \rightarrow 0$ as $\lambda \rightarrow \infty$. It suffices to choose a sequence $\left\{\lambda_{j}\right\}_{j=1}^{\infty}$ such that $C\left(\lambda_{j}\right)<2^{-j}$ and $\left\|b_{k_{j}^{\prime}}-b\right\|<\lambda_{j}^{-1} 2^{-2 j}$.

Choose $n_{j}$ such that for $N>n_{j}$,

$$
\tau\left(\left\{\left|A_{N}\left(b_{k_{j}^{\prime}}\right)-x_{j}\right|>2^{-j}\right\}\right)<2^{-j} .
$$

This is possible since $A_{n}\left(b_{k_{j}^{\prime}}\right)$ converges stochastically to $x_{j}$. Then for $j, i \in \mathbb{N}$ and $n>n_{i+j}$,

$$
\begin{aligned}
& \tau\left(\left\{\left|x_{j}-x_{j+i}\right|>3 \cdot 2^{-j}\right\}\right) \\
& =\tau\left(\left\{\mid\left(x_{j}-A_{n}\left(b_{k_{j}^{\prime}}\right)\right)+\left(A_{n}\left(b_{k_{j}^{\prime}}\right)-A_{n}\left(b_{k_{j+i}^{\prime}}\right)\right)\right.\right. \\
& \left.\left.\quad+\left(A_{n}\left(b_{k_{j+i}^{\prime}}\right)-x_{j+i}\right) \mid>3 \cdot 2^{-j}\right\}\right) \\
& \leq \tau\left(\left\{\left|A_{n}\left(b_{k_{j}^{\prime}}\right)-x_{j}\right|>2^{-j}\right\}\right)+\tau\left(\left\{\left|A_{n}\left(b_{k_{j}^{\prime}}\right)-A_{n}\left(b_{k_{j+i}^{\prime}}\right)\right|>2^{-j}\right\}\right) \\
& \quad+\tau\left(\left\{\left|A_{n}\left(b_{k_{j+i}^{\prime}}\right)-x_{j+i}\right|>2^{-(j+i)}\right\}\right) \\
& \leq 3 \cdot 2^{-j} .
\end{aligned}
$$

Here the first inequality follows from (3).

Denote the stochastic limit of $\left\{x_{j}\right\}_{j=1}^{\infty}$ by $x_{0}$. Taking a subsequence of $\left\{x_{j}\right\}$ if necessary, we suppose that

$$
\tau\left(\left\{\left|x_{j}-x_{0}\right|>2^{-j}\right\}\right) \leq 2^{-j}
$$

The sequence $\left\{A_{n}(b)\right\}_{n=1}^{\infty}$ converges to $x_{0}$ stochastically. Indeed, for $n>n_{j}$,

$$
\begin{aligned}
& \tau\left(\left\{\left|A_{n}(b)-x_{0}\right|>3 \cdot 2^{-j}\right\}\right) \\
&= \tau\left(\left\{\left|\left(A_{n}(b)-A_{n}\left(b_{k_{j}^{\prime}}\right)\right)+\left(A_{n}\left(b_{k_{j}^{\prime}}\right)-x_{j}\right)+\left(x_{j}-x_{0}\right)\right|>3 \cdot 2^{-j}\right\}\right) \\
& \leq \tau\left(\left\{\left|A_{n}(b)-A_{n}\left(b_{k_{j}^{\prime}}\right)\right|>2^{-j}\right\}\right)+\tau\left(\left\{\left|A_{n}\left(b_{k_{j}^{\prime}}\right)-x_{j}\right|>2^{-j}\right\}\right) \\
& \quad+\tau\left(\left\{\left|x_{j}-x_{0}\right|>2^{-j}\right\}\right) \\
& \leq 3 \cdot 2^{-j} .
\end{aligned}
$$

Here the first inequality follows from (3) and the second follows by noting that the first part follows from (16), the second from (17) and the choice of $n$, and the third from (19). Part (i) is thus established.

(ii) Suppose that for every $b \in B$ and $\lambda \in \mathbb{R}_{+}$,

$$
\sup _{n} \tau\left(\left\{\left|A_{n}(b)\right|>\lambda\right\}\right) \stackrel{\lambda \rightarrow \infty}{\longrightarrow} 0 .
$$


For fixed $\varepsilon>0$ and $\lambda \in \mathbb{N}$, define $B_{\lambda}=\left\{b \in B: \sup _{n} \tau\left(\left\{\left|A_{n}(b)\right|>\lambda\right\}\right) \leq \varepsilon\right\}$. Then from (21) it follows that

$$
B=\bigcup_{\lambda \in \mathbb{N}} B_{\lambda} .
$$

Let $B_{\lambda, k}:=\left\{b \in B: \sup _{n \geq k} \tau\left(\left\{\left|A_{n}(b)\right|>\lambda\right\}\right) \leq \varepsilon\right\}$. Then

$$
B_{\lambda}=\bigcap_{k \in \mathbb{N}} B_{\lambda, k}
$$

The sets $B_{\lambda, k}$ are closed. Indeed, let $B_{\lambda, k} \supset\left\{b_{j}\right\}_{j=1}^{\infty}$ converge to $b \in B$. Then

$$
\begin{aligned}
& \tau\left(\left\{\left|A_{n}(b)\right|>\lambda+\gamma\right\}\right)=\tau\left(\left\{\left|A_{n}\left(b_{j}\right)-\left(A_{n}\left(b_{j}\right)-A_{n}(b)\right)\right|>\lambda+\gamma\right\}\right) \\
& \leq \tau\left(\left\{\left|A_{n}\left(b_{j}\right)\right|>\lambda\right\}\right)+\tau\left(\left\{\left|\left(A_{n}\left(b_{j}\right)-A_{n}(b)\right)\right|>\gamma\right\}\right) \leq \varepsilon .
\end{aligned}
$$

Here the first inequality follows from (3). The estimate of the first term follows from the definition of $B_{\lambda, k}$, while that of the second term becomes valid for sufficiently large $j$, and follows from the free choice of $b_{j}$ and from the continuity of $A_{n}$ in measure.

Since $\lambda_{t}(x)$ is continuous from the right due to Remark 1.3, we have

$$
\tau\left(\left\{\left|A_{n}(b)\right|>\lambda\right\}\right)=\lim _{m \rightarrow \infty} \tau\left(\left\{\left|A_{n}(b)\right|>\lambda+\gamma_{m}\right\}\right) \leq \varepsilon
$$

where $\gamma_{m} \rightarrow 0$ as $m \rightarrow \infty$. Hence, $b \in B_{\lambda, k}$, so $B_{\lambda, k}$ is closed. Then $B_{\lambda}$ is closed as an intersection of closed sets.

It follows from the Baire category principle that there exists $\lambda$ such that $B_{\lambda}$ has a non-empty interior. Let $B\left(b_{0}, r\right)=\left\{b \in B:\left\|b-b_{0}\right\| \leq r\right\} \subset B_{\lambda}$. Then

$$
\tau\left(\left\{\left|A_{n}(b)\right|>\lambda\right\}\right) \leq \varepsilon \quad \text { for every } b \in B\left(b_{0}, r\right) .
$$

Moreover, for $b=b_{0}-r c \in B\left(b_{0}, r\right)$ with $c \in B,\|c\| \leq 1$, we have

$$
\begin{aligned}
\tau\left(\left\{\left|A_{n}(r c)\right|>2 \lambda\right\}\right) & =\tau\left(\left\{\left|A_{n}\left(r c-b_{0}\right)+A_{n}\left(b_{0}\right)\right|>2 \lambda\right\}\right) \\
& \leq \tau\left(\left\{\left|A_{n}\left(r c-b_{0}\right)\right|>\lambda\right\}\right)+\tau\left(\left\{\left|A_{n}\left(b_{0}\right)\right|>\lambda\right\}\right) \\
& \leq 2 \varepsilon .
\end{aligned}
$$

Let $\gamma \geq 2 \lambda / r$. From (27) it follows that $\tau\left(\left\{\left|A_{n}(c)\right|>\gamma\right\}\right) \leq 2 \varepsilon$ for every $c \in B$ with $\|c\| \leq 1$. that

Let $C(\gamma)=\sup _{c \in B,\|c\| \leq 1} \tau\left(\left\{\left|A_{n}(c)\right|>\gamma\right\}\right) \leq 2 \varepsilon$. Free choice of $\varepsilon$ implies

$$
\lim _{\gamma \rightarrow \infty} C(\gamma)=0
$$

hence (11) is valid.

For application of Theorem 2.3, it is convenient to combine (i) and (ii). 
Theorem 2.4. Let $(B,\|\cdot\|)$ be a Banach space, $A_{n}$ a set of continuousin-measure linear maps from $B$ into $S(M), \lambda \in \mathbb{R}_{+}$, and suppose that for each $b \in B$,

$$
\lim _{\lambda \rightarrow \infty} \sup _{n \in \mathbb{N}} \tau\left(\left\{\left|A_{n}(b)\right|>\lambda\right\}\right)=0 .
$$

Then the subset $\widetilde{B}$ of $B$ where $A_{n}(b)$ converges in measure is closed in $B$.

Proof. Follows immediately by applying consecutively (ii) and (i) of Theorem 2.3 .

Let $e$ be a projection in $M$, and let $M_{e}$ be the von Neumann algebra consisting of the operators of the form exe, $x \in M$ (we denote exe by $x_{e}$ ). If $\tau$ is a semifinite normal faithful trace on $M$, then $\tau_{e}=\left.\tau\right|_{M_{e}}$ is a semifinite (possibly finite) faithful normal trace on $M_{e}$. Indeed, the tracial property, semifiniteness, normality and faithfulness of $\tau_{e}$ follow directly from similar properties of $\tau$. The space $S\left(M_{e}, \tau_{e}\right)$ is isomorphic to $S(M, \tau)_{e}$ since both are closures of $\left(M_{\tau \text {-finite support }}\right)_{e}=\left(M_{e}\right)_{\tau_{e} \text {-finite support }}$.

Proposition 2.5. Let $B_{n}$ be a sequence of continuous-in-measure operators on $S(M, \tau)$, and $e_{i} \in P(M), i=1,2$, with $\mathbb{I}=e_{1}+e_{2}$. Suppose that $e_{i}\left(B_{n}(x)\right)=B_{n}\left(x_{e_{i}}\right)=\left(B_{n}(x)\right) e_{i}$ for every $n \in \mathbb{N}$ and $x \in S(M, \tau)$, or in other words $e_{i}$ commutes with $B_{n}$. Suppose also that

$$
\lim _{\lambda \rightarrow \infty} \sup _{n \in \mathbb{N}} \tau\left(\left\{\left|B_{n}\left(x_{e_{i}}\right)\right|>\lambda\right\}\right)=0 \quad \text { for } i=1,2 \text { and all } x \in S(M, \tau) .
$$

Then

$$
\lim _{\lambda \rightarrow \infty} \sup _{n \in \mathbb{N}} \tau\left(\left\{\left|B_{n}(x)\right|>\lambda\right\}\right)=0 .
$$

Proof. We have

$$
\tau\left(\left\{\left|B_{n}\left(x_{e_{i}}\right)\right|>\lambda\right\}\right)=\tau\left(e_{i}\left\{\left|B_{n}(x)\right|>\lambda\right\}\right) .
$$

Indeed, since for $x \in S_{h}(M)$ (the set of all self-adjoint operators in $S(M)$ ) we have $\tau(\{|x|>\lambda\}) \rightarrow 0$ as $\lambda \rightarrow \infty$, there is a sequence of polynomials $P_{j}(y)$ in $\mathbb{R}$ converging to $\chi_{\{|y|>\lambda\}}(y)$ pointwise such that $P_{j}(x)$ converges to $\chi_{\{|x|>\lambda\}}(x)$ stochastically. Then by [6, Proposition 3.2],

$$
\begin{aligned}
& \tau\left(\left\{\left|B_{n}\left(x_{e_{i}}\right)\right|>\lambda\right\}\right)=\lim _{j} \tau\left(P_{j}\left(B_{n}\left(x_{e_{i}}\right)\right)\right)=\lim _{j} \tau\left(P_{j}\left(B_{n}\left(e_{i} x e_{i}\right)\right)\right) \\
& =\lim _{j} \tau\left(P_{j}\left(e_{i} B_{n}(x) e_{i}\right)\right)=\lim _{j} \tau\left(e_{i} P_{j}\left(B_{n}(x)\right)\right)=\tau\left(e_{i}\left\{\left|B_{n}(x)\right|>\lambda\right\}\right) .
\end{aligned}
$$

Statement (31) now follows from the fact that (as $B_{n}$ commutes with $e_{i}$ and (3) holds)

$$
\begin{aligned}
\tau\left(\left\{\left|B_{n}(x)\right|>\lambda_{1}\right.\right. & \left.\left.+\lambda_{2}\right\}\right)=\tau\left(\left\{\left|\left(e_{1}+e_{2}\right) B_{n}(x)\left(e_{1}+e_{2}\right)\right|>\lambda_{1}+\lambda_{2}\right\}\right) \\
& =\tau\left(\left\{\left|e_{1} B_{n}(x) e_{1}+e_{2} B_{n}(x) e_{2}\right|>\lambda_{1}+\lambda_{2}\right\}\right) \\
& \leq \tau\left(\left\{\left|B_{n}\left(x_{e_{1}}\right)\right|>\lambda_{1}\right\}\right)+\tau\left(\left\{\left|B_{n}\left(x_{e_{2}}\right)\right|>\lambda_{2}\right\}\right) .
\end{aligned}
$$


REMARK 2.1. We are going to use (29) in the next section when dealing with stochastic ergodic theorems, since it holds in that setting.

3. Stochastic ergodic theorems. In this section we establish stochastic convergence of bounded Besicovitch sequences, and present stochastic ergodic theorems for uniform subsequences.

Here we use the following assumptions: $M$ is a von Neumann algebra with a faithful normal tracial state $\tau$, and $\alpha$ a $*$-automorphism of the algebra $M$. Set $A_{n}(x)=n^{-1} \sum_{l=1}^{n-1} \alpha^{l}(x)$ for $x \in M$. Define $\alpha^{\prime}$ as a linear map on $L_{1}(M, \tau)$ satisfying $\tau(x \cdot \alpha(y))=\tau\left(\alpha^{\prime}(x) y\right)$ for $x \in L_{1}(M, \tau), y \in M$, and $A_{n}^{\prime}(x)=n^{-1} \sum_{l=1}^{n-1}\left(\alpha^{\prime}\right)^{l}(x)$, for $x \in L_{1}(M, \tau)$.

Let us also recall some definitions from Grabarnik and Katz [10] and from Chilin, Litvinov and Skalski [2].

Definition 3.1. A positive operator $h \in M_{+}$is called weakly wandering if

$$
\left\|A_{n}(h)\right\|_{\infty} \stackrel{n \rightarrow \infty}{\longrightarrow} 0
$$

The following definition comes from [18], [22] (see also [2]).

DeFinition 3.2. A sequence $\left\{x_{n}\right\}_{n=1}^{\infty}$ converges to $x_{0}$ bilaterally almost uniformly (b.a.u.) in $S(M)$ if for every $\delta>0$ there exists a projection $e \in$ $P(M)$ such that $\left\|\left(x_{n}-x\right) e\right\|_{\infty} \rightarrow 0$ and $\tau(\mathbb{I}-e)<\delta$.

REMARK 3.1. Note that b.a.u. convergence implies stochastic convergence.

The following definition is due to Ryll-Nardzewski [16].

Definition 3.3. Let $\mathbb{C}_{1}$ denote the unit circle in $\mathbb{C}$. A trigonometric polynomial is a map $P_{k}: \mathbb{N} \rightarrow \mathbb{C}$, where $P_{k}(n)=\sum_{j=0}^{k-1} b_{j} \lambda_{j}^{n}$ for some $\left\{\lambda_{j}\right\}_{j=0}^{k-1} \subset \mathbb{C}_{1}$ and $\left\{b_{j}\right\}_{j=0}^{k-1} \subset \mathbb{R}$.

Bounded Besicovitch sequences are bounded sequences from the $l_{1}$-average closure of trigonometric polynomials.

More precisely,

Definition 3.4. A sequence $\beta_{n}$ of complex numbers is called a bounded Besicovitch sequence if

(i) $\left|\beta_{n}\right| \leq C<\infty$ for every $n \in \mathbb{N}$,

(ii) for every $\varepsilon>0$, there exists a trigonometric polynomial $P_{k}$ such that

$$
\limsup _{n} \frac{1}{n} \sum_{j=1}^{n-1}\left|\beta_{j}-P_{k}(j)\right|<\varepsilon .
$$

Let $\mu$ be a normalized Lebesgue measure (Radon measure) on $\mathbb{C}_{1}$ and $\widetilde{M}$ be the von Neumann algebra of all essentially bounded ultra-weakly 
measurable functions $f:\left(\mathbb{C}_{1}, \mu\right) \rightarrow M$. The algebra $\widetilde{M}$ is isomorphic to $L_{\infty}\left(\mathbb{C}_{1}, \mu\right) \bar{\otimes} M$, the $W^{*}$ tensor product of $L_{\infty}\left(\mathbb{C}_{1}, \mu\right)$ and $M$, and a dual to the space $L_{1}\left(\mathbb{C}_{1}, \mu\right) \bar{\otimes} M_{*}$ (for the definition of $W^{*}$ tensor product and the form of its predual space, see for example Takesaki [20, Theorem IV.7.17]). The space $L_{1}\left(\mathbb{C}_{1}, \mu\right) \bar{\otimes} M_{*}$ can be considered as a set of $L_{1}$ functions on $\left(\mathbb{C}_{1}, \mu\right)$ with values in $M_{*}$. The algebra $\widetilde{M}$ also has a natural trace $\widetilde{\tau}(f)=$ $\int_{\mathbb{C}_{1}} \tau(f(z)) d \mu(z)$, and $\widetilde{M}_{*}$ is isomorphic to $L_{1}(\widetilde{M}, \widetilde{\tau})$.

Let $\sigma$ be an automorphism of $\left(\mathbb{C}_{1}, \mu\right)$ as a Lebesgue space with measure. We define the automorphism $\alpha \otimes \sigma$ of $(\widetilde{M}, \widetilde{\tau})$ as the closure of the linear extension of the automorphism acting on $(\widetilde{M}, \widetilde{\tau}) \ni x(z)$ as $\alpha \otimes \sigma(x(z))=$ $\alpha(x(\sigma(z)))$.

EXAmple 3.1. An example of such an automorphism is $\widetilde{\alpha}_{\lambda}(x(z))=$ $\alpha(x(\lambda z)))$ for $\lambda \in \mathbb{C}_{1}$. In this case

$$
A_{n}(x)=\frac{1}{n} \sum_{l=1}^{n-1} \widetilde{\alpha}_{\lambda}^{l}(x)=\frac{1}{n} \sum_{l=1}^{n-1} \alpha^{l}\left(x\left(\lambda^{l} z\right)\right) .
$$

In particular, if $x(z) \equiv z x$ for $x \in M$ then

$$
A_{n}(x z)=z \frac{1}{n} \sum_{l=1}^{n-1} \lambda^{l} \alpha^{l}(x) .
$$

The following lemma connects stochastic convergence in $L_{1}(\widetilde{M}, \widetilde{\tau})$ with pointwise convergence on $C_{1}$ and stochastic convergence in $M$ (cf. [2]).

LEMMA 3.2.

(i) If $L_{1}(\widetilde{M}, \widetilde{\tau}) \ni x_{n} \rightarrow x_{0} \in L_{1}(\widetilde{M}, \widetilde{\tau})$ b.a.u. as $n \rightarrow \infty$, then $x_{n}(z) \rightarrow$ $x_{0}(z)$ stochastically for almost every $z \in \mathbb{C}_{1}$.

(ii) Suppose that $h$ is a weakly wandering operator with $\operatorname{supp}(h)=\mathbb{I}$ for a sequence $A_{n}$. Then $A_{n}^{\prime}(x)$ converges to 0 stochastically.

(iii) Let $\mathcal{N}=(M, \tau) \bar{\otimes} L_{\infty}(X, \mu)$ (X being a separable Hausdorff compact set, and $\mu$ a Lebesgue measure), $\alpha$ an automorphism of $M$, and $\sigma$ an automorphism of $L_{\infty}(X, \mu)$. Then $\alpha \otimes \sigma$ is an automorphism of $\mathcal{N}$. Suppose that $h$ is a weakly wandering operator with $\operatorname{supp}(h)=\mathbb{I}$ for a sequence $A_{n}$ corresponding to the automorphism $\alpha \otimes \sigma$. Then $A_{n}^{\prime}(x(z))$ converges to 0 stochastically for almost every $z \in X$.

Proof. By [2, Lemma 4.1], the hypothesis of part (i) implies b.a.u. convergence of $x_{n}(z)$ to $x_{0}(z)$ for almost every $z$ in $\mathbb{C}_{1}$. Hence bilateral stochastic convergence holds. This implies the statement of (i), since bilateral stochastic convergence is equivalent to (one-sided) stochastic convergence (see [2, Theorem 2.2]).

(ii) Suppose that $x \in L_{1}(M, \tau)_{+}$and $A_{n}^{\prime}(x)$ is a sequence satisfying

$$
\tau\left(A_{n}^{\prime}(x) h\right) \rightarrow 0 \quad \text { as } n \rightarrow \infty .
$$


Then

$$
t s \cdot \tau\left(\left\{A_{n}^{\prime}(x)>t\right\} \wedge\{h>s\}\right) \leq \tau\left(A_{n}^{\prime}(x) h\right) .
$$

Indeed, for any $e_{1}, e_{2} \in P(M)$, we have $e_{1} e_{2} e_{1} \geq e_{1} \wedge e_{2}$. To see this, note that since $e_{1} \wedge e_{2}$ commutes with $e_{1}, e_{2}$, we have $\left(\mathbb{I}-e_{1} \wedge e_{2}\right) e_{1} e_{2} e_{1}\left(e_{1} \wedge e_{2}\right)=0$, hence

$$
\begin{aligned}
e_{1} e_{2} e_{1} & =\left(\mathbb{I}-e_{1} \wedge e_{2}\right) e_{1} e_{2} e_{1}\left(\mathbb{I}-e_{1} \wedge e_{2}\right)+\left(e_{1} \wedge e_{2}\right) e_{1} e_{2} e_{1}\left(e_{1} \wedge e_{2}\right) \\
& =\left(\mathbb{I}-e_{1} \wedge e_{2}\right) e_{1} e_{2} e_{1}\left(\mathbb{I}-e_{1} \wedge e_{2}\right)+\left(e_{1} \wedge e_{2}\right) .
\end{aligned}
$$

Thus,

$$
\begin{aligned}
& t s \cdot \tau\left(\left\{A_{n}^{\prime}(x)>t\right\} \wedge\{h>s\}\right) \leq t \tau\left(\left\{A_{n}^{\prime}(x)>t\right\} s\{h>s\}\left\{A_{n}^{\prime}(x)>t\right\}\right) \\
& \leq t \tau\left(\left\{A_{n}^{\prime}(x)>t\right\} h\left\{A_{n}^{\prime}(x)>t\right\}\right)=t \tau\left(\left\{A_{n}^{\prime}(x)>t\right\} h\right) \leq \tau\left(A_{n}^{\prime}(x) h\right) .
\end{aligned}
$$

Hence, (40) is valid.

Furthermore,

$$
\tau\left(\left\{A_{n}^{\prime}(x)>t\right\}\right) \leq \frac{1}{t s} \tau\left(A_{n}^{\prime}(x) h\right)+\tau(\mathbb{I}-\{h>s\}) .
$$

This follows from (40), and from the fact that $\tau\left(e_{1}\right) \leq \tau\left(e_{1} \wedge e_{2}\right)+\tau\left(\mathbb{I}-e_{2}\right)$. Indeed,

$$
\begin{aligned}
\tau\left(e_{1}-e_{1} \wedge e_{2}\right) & =\tau\left(e_{1}\left(\mathbb{I}-e_{1} \wedge e_{2}\right)\right)=\tau\left(e_{1}\left(\mathbb{I}-e_{1} \wedge e_{2}\right) e_{1}\right) \\
& \leq \tau\left(e_{1}\left(\mathbb{I}-e_{2}\right) e_{1}\right)=\tau\left(e_{1}\left(\mathbb{I}-e_{2}\right)\right) \leq \tau\left(\mathbb{I}-e_{2}\right) .
\end{aligned}
$$

Hence, (42) is valid.

Note that inequality (42) together with the fact that $\tau\left(A_{n}^{\prime}(x) h\right) \rightarrow 0$ as $n \rightarrow \infty$ implies that $\sup _{n \in \mathbb{N}} \tau\left(\left\{\left|A_{n}(b)\right|>\lambda\|b\|\right\}\right) \leq C(\lambda)$. Indeed, the sequence $\left\{\tau\left(A_{n}^{\prime}(x) h\right)\right\}_{n=1}^{\infty}$, being convergent, is bounded by a constant $C_{0}$. Choose a decreasing sequence $\left\{s_{j}\right\}_{j=1}^{\infty} \subset \mathbb{R}_{+}$such that $\tau\left(\mathbb{I}-\left\{h>s_{j}\right\}\right)<2^{-j}$, and set $t_{j}=2^{j} s_{j}^{-1}$. Then

$$
\tau\left(\left\{A_{n}^{\prime}(x)>t_{j}\right\}\right) \leq \frac{1}{t_{j} s_{j}} C_{0}+2^{-j}=\left(C_{0}+1\right) 2^{-j} .
$$

Hence Theorem 2.4 implies the stochastic convergence of $A_{n}^{\prime}(x)$. Indeed, for a dense subset in $L_{1}(M, \tau)$ of elements of the form $\widehat{x}=x-A_{k}^{\prime}(x)+\widetilde{x}$ (here $x \in M \cap L_{1}(M, \tau)$ and $\widetilde{x} \in M$ is an $\alpha^{\prime}$-invariant element, see [13, Theorem 1.5(iii), p. 273]), $A_{n}^{\prime}(\widehat{x})$ converges in $L_{1}$, hence stochastically.

(iii) The proof is along the same lines as for (ii), with the necessary adjustments. Let $E_{1}$ be a conditional expectation with respect to the trace $\tau \otimes \mu$ of $(M, \tau) \bar{\otimes} L_{\infty}(X, \mu)$ onto $(M, \tau) \bar{\otimes} \operatorname{Const}(X, \mu)$, and $E_{2}$ a conditional expectation with respect to the trace $\tau \otimes \mu$ of $(M, \tau) \bar{\otimes} L_{\infty}(X, \mu)$ onto $\mathbb{C} \cdot \mathbb{I} \bar{\otimes}$ $L_{\infty}(X, \mu)$ (for the definition of the first conditional expectation, see [20]). Due to the form of $\alpha \otimes \sigma$, both $E_{j}$ 's commute with $A_{n}$, for $j=1,2$. 
Since

$$
\left\|A_{n}(h)\right\|_{\infty} \geq\left\|E_{1} A_{n}(h)\right\|_{\infty}=\left\|A_{n}\left(E_{1} h\right)\right\|_{\infty},
$$

and $\operatorname{supp}(h) \leq \operatorname{supp}\left(E_{1} h\right)$, it follows that $\operatorname{supp}\left(E_{1} h\right)=\mathbb{I}$. Indeed, $x \geq 0$, $x \neq 0$ implies $\tau\left(E_{1} x\right)=\tau(x)>0$, hence $0<\tau\left(\left(E_{1} a\right) h\right)=\tau\left(a\left(E_{1} h\right)\right)$ and $\operatorname{supp}\left(E_{1} h\right)=\mathbb{I}$ for every $a \in M$.

Hence $E_{1}(h)$ is a weakly wandering operator.

For positive $x(z) \in L_{1}(M, \tau) \bar{\otimes} L_{1}(X, \mu)$ we have

$$
\|x\|_{1}=\int_{X}\|x(z)\|_{1} d \mu(z)
$$

hence $\|x(z)\|_{1}$ is an $L_{1}(X, \mu)$ function. Applying the classical Hopf inequality (see for example [13, Theorem 2.1, p. 8]), we get

$$
\mu\left(\sup _{n}\left\{\left\|A_{n}^{\prime}(x)(z)\right\|_{1}>\lambda\right\}\right) \leq \frac{\text { Const }}{\lambda} \int_{X}\|x(z)\|_{1} d \mu(z),
$$

so outside a set $X_{0} \subset X$ of small measure the value of $\left\|A_{n}^{\prime}(x)(z)\right\|_{1}$ is uniformly bounded. Proceeding as in part (ii) applied to every $z \in X_{0}$, we get stochastic convergence for every $z \in X_{0}$.

TheOREM 3.3 (Neveu decomposition for special tensor products of von Neumann algebras). Let $\mathcal{N}=(M, \tau) \bar{\otimes} L_{\infty}(X, \mu)$ (X being a Hausdorff separable compact set, and $\mu$ a Lebesgue measure), $\alpha$ an automorphism of $M$, and $\sigma$ an automorphism of $L_{\infty}(X, \mu)$. Then $\widetilde{\alpha}=\alpha \otimes \sigma$ is an automorphism of $\mathcal{N}$. Suppose that, in addition, the automorphism $\sigma$ is ergodic. Then there exists an $\widetilde{\alpha}$-invariant projection in $\mathcal{N}$ of the form $e_{1}=e_{11} \otimes \mathbb{I}$, with $e_{1}(z)$ $=e_{M}$ for almost every $z \in X$, such that, setting $e_{2}=\mathbb{I}-e_{1}$, we have:

(i) There exists a normal state $\varrho$ on $\mathcal{N}$ with $\operatorname{supp}(\varrho)=e_{1}$, and for almost each $z \in X, \varrho(z)$ is invariant with respect to $\alpha^{\prime}$.

(ii) There exists a weakly wandering operator $h \in \mathcal{N}$ with $\operatorname{supp}(h)=e_{2}$ and for almost each $z \in X, h(z)$ is a weakly wandering operator in $M$.

Proof. Corollary 1.1 of [10] implies existence of a projection $\widetilde{e}_{1}$ in $\mathcal{N}$ such that $(\mathrm{i})$ there exists an $\widetilde{\alpha}^{\prime}$-invariant normal state $\varrho$ with $\operatorname{supp}(\varrho)=\widetilde{e}_{1}$ and (ii) there exists a weakly wandering operator $h \in \mathcal{N}$ with support $\mathbb{I}-\widetilde{e}_{1}$. Our goal is to show that similar statements are valid for almost every $z \in X$.

Since $\sigma$ is ergodic, for every $x \in M \otimes \operatorname{Const}(X, \mu)$ (here $M \otimes \operatorname{Const}(X, \mu)$ is the space of constant functions on $X$ with values in $M$ ) we have

$$
\begin{aligned}
\varrho(z)(x(z)) & =\left(\widetilde{\alpha}^{\prime} \varrho(z)\right)(x(z))=\varrho(z)(\alpha(x(\sigma(z)))) \\
& =\varrho(z)(\alpha(x(z)))=\left(\alpha^{\prime}(\varrho(z))\right)(x(z)),
\end{aligned}
$$

that is, $\varrho(z)$ is $\alpha^{\prime}$-invariant. Suppose that $z \mapsto \varrho(z)$ is not constant, so there exists $r_{0} \in \mathbb{R}_{+}$and $x(z) \equiv x_{0} \in M_{+}$with $\mu\left(\left\{z \in X: \varrho(z)(x(z)) \leq r_{0}\right\}\right)>0$ 
and $\mu\left(\left\{z \in X: \varrho(z)(x(z))<r_{0}\right\}\right)>0$. Since $\sigma$ is ergodic there exists $n \in \mathbb{N}$ such that

$$
\mu\left(\sigma^{-n}\left(\left\{z \in X: \varrho(z)(x(z)) \leq r_{0}\right\}\right) \cap\left\{z \in X: \varrho(z)(x(z))<r_{0}\right\}\right)>0 .
$$

Hence,

$$
\begin{aligned}
\varrho(z)(x(z)) & =\left(\widetilde{\alpha}^{\prime n} \varrho(z)\right)(x(z))=(\alpha)^{\prime n}(\varrho(z))\left(x\left(\sigma^{n}(z)\right)\right) \\
& =\varrho(z)\left(x\left(\sigma^{n}(z)\right)\right)=\varrho\left(\sigma^{-n} z\right)(x((z))),
\end{aligned}
$$

so $r_{0} \geq \varrho(z)\left(x_{0}\right)=\varrho\left(\sigma^{-n} z\right)\left(x_{0}\right)<r_{0}$. The contradiction shows that $z \mapsto \varrho(z)$ is constant.

This implies that $\operatorname{supp}(\varrho)=\operatorname{supp}(\varrho(z))=\widetilde{e}_{1}(z)$ is constant.

The proof of (ii) follows the reasoning for (45).

THEOREM 3.4. Let $\mathcal{N}=(M, \tau) \bar{\otimes} L_{\infty}(X, \mu)$ (X being a separable Hausdorf compact set, and $\mu$ a normalized Lebesgue measure), $\alpha$ an automorphism of $M$, and $\sigma$ an automorphism of $L_{\infty}(X, \mu)$. Then $\widetilde{\alpha}=\alpha \otimes \sigma$ is an automorphism of $\mathcal{N}$. Suppose that, in addition, $\sigma$ is ergodic. Then for almost every $z \in X$, the averages $A_{n}^{\prime}(x(z))$ converge stochastically.

Proof. This follows directly by applying Theorems 3.3 and 3.2 to the part of the partition where there exists a weakly wandering operator, and by applying the regular individual ergodic theorem [22] to the part where an invariant normal state exists $(3.3(\mathrm{i}))$.

Now we are in a position to prove stochastic convergence of bounded Besicovitch sequences.

TheOREM 3.5 (Stochastic ergodic theorem for bounded Besicovitch sequences). Let $\left\{\beta_{j}\right\}_{j=1}^{\infty}$ be a bounded Besicovitch sequence, and $M$ a von Neumann algebra with a finite faithful normal tracial state $\tau$. Let $\alpha$ be an automorphism of $M$. Then the sequence

$$
\widetilde{A}_{n}(x)=\frac{1}{n} \sum_{j=0}^{n-1} \beta_{j}\left(\alpha^{\prime}\right)^{j}(x)
$$

converges stochastically for $x \in L_{1}(M, \tau)$.

Proof. Suppose first that $\left\{\beta_{j}\right\}=\left\{P_{k}(j)\right\}$ for a trigonometric polynomial $P_{k}(j)$. Then the statement of the theorem is valid.

Indeed, choosing $\widetilde{\alpha}$ as in Example 3.1, we deduce from Theorem 2.4 and from the fact that every irrational rotation on $\mathbb{C}_{1}$ is ergodic (the equidistribution Kronecker-Weyl theorem, see e.g. [12, p. 146]) that

$$
A_{n}(x z)=z \frac{1}{n} \sum_{l=1}^{n-1} \lambda^{l} \alpha^{l}(x)
$$

hence 


$$
\frac{1}{n} \sum_{l=1}^{n-1} \lambda^{l}\left(\alpha^{\prime}\right)^{l}(x)
$$

converges stochastically for irrational $\lambda$.

For rational $\lambda$, the convergence follows from the fact that (51) is a finite combination of averages of $\left(\alpha^{\prime}\right)^{m}$, where $m$ is the denominator.

Taking linear combinations of terms as in (51) implies the statement for trigonometric polynomials.

Next, the statement of the theorem is valid for $x \in M \cap S(M)$. Indeed, approximating the bounded Besicovitch sequence by trigonometric polynomials as in (36), we get for $A_{n}(k, x)=n^{-1} \sum_{l=1}^{n-1} P_{k}(l) \cdot\left(\alpha^{\prime}\right)^{l}(x)$,

$$
\left\|\widetilde{A}_{n}(x)-A_{n}(k, x)\right\|_{\infty} \leq \frac{1}{n}\left(\sum_{l=0}^{n-1}\left|\beta_{l}-P_{k}(l)\right|\right)\|x\|_{\infty}
$$

which gives stochastic convergence.

Note also that for every $x \in L_{1}(M, \tau)$

$$
\left\|\widetilde{A}_{n}(x)-A_{n}(k, x)\right\|_{1} \leq \frac{1}{n}\left(\sum_{l=0}^{n-1}\left|\beta_{l}-P_{k}(l)\right|\right)\|x\|_{1} .
$$

Hence by Remark 2.1 the averages $\widetilde{A}_{n}(x)$ are uniformly bounded in the sense of (11).

The assertion of the theorem follows from the stochastic Banach principle, Theorem 2.4 and density of $M \cap S(M)$ in $L_{1}(M, \tau)$.

The next theorem is a consequence of the stochastic ergodic theorem for bounded Besicovitch sequences (cf. [14]). (For the definitions below see for example [13, p. 260]).

Let $\sigma$ be a homeomorphism of a compact metric space $X$ with metric $\varrho$ such that all powers of $\sigma^{l}$ are equicontinuous. Assume also that there exists $z \in X$ with dense orbit $\sigma^{l}(z)$ in $X$. Then there exists a unique (hence ergodic) $\sigma$-invariant measure $\nu$ on the $\sigma$-algebra of Borel sets $\mathfrak{B}$. Each non-empty open set has a positive $\nu$ measure.

A sequence $u_{j}$ is called uniform if there exists a dynamical system $(X, \mathfrak{B}, \nu, \sigma)$, a set $Y \in \mathfrak{B}$ with $\nu(\partial Y)=0$ and $\nu(Y)>0$, and a point $y \in X$ such that $u_{j}$ is the $j$ th entry time of the orbit of $y$ into $Y$.

THEOREM 3.6. Let $M, \tau, \alpha$ be as in the preceding theorem, and $\left\{u_{j}\right\}_{j \geq 0}$ a uniform sequence. Then the averages

$$
\frac{1}{n} \sum_{j=0}^{n-1}\left(\alpha^{\prime}\right)^{u_{j}} x
$$

converge stochastically for $x \in L_{1}(M, \tau)$. 
Proof. The proof is along the same lines as for [16] or [14, Theorem 4], and we give its outline with the necessary adjustments. For $x \in L_{1}(M, \tau) \cap M$ we use the preceding theorem to show stochastic convergence. In passing from $x \in L_{1}(M, \tau) \cap M$ to $x \in L_{1}(M, \tau)$, we use the Neveu decomposition 3.3 to distinguish between two cases. Applicability of the stochastic Banach principle to the part of the partition (3.3(ii)) with weakly wandering operator follows from Lemma 3.2(ii). This implies stochastic convergence on this part of the Neveu decomposition. The regular individual ergodic theorem [22] applied to the part of the Neveu partition (3.3(i)) where an invariant normal state exists implies stochastic convergence on this part of the Neveu decomposition.

This completes the outline of the proof.

REMARK 3.2. Similar results hold for the case where $M$ is a semifinite JBW algebra with faithful normal trace $\tau$.

\section{References}

[1] O. Bratteli and D. Robinson, Operator Algebras and Quantum Statistical Mechanics, Vol. I, Texts Monogr. Phys., Springer, New York, 1979.

[2] V. I. Chilin, S. Litvinov and A. Skalski, A few remarks in non-commutative ergodic theory, J. Operator Theory 53 (2005), 331-350.

[3] J. Dixmier, Formes linéaires sur un anneau d'opérateurs, Bull. Soc. Math. France 81 (1953), 9-39.

[4] -, Les algèbres d'opérateurs dans l'espace hilbertien (algèbres de von Neumann), Gauthier-Villars, Paris, 1960.

[5] N. Dunford and J. T. Schwartz, Linear Operators I, Pure Appl. Math. 7, Interscience, New York, 1958.

[6] T. Fack and H. Kosaki, Generalized s-numbers of $\tau$-measurable operators, Pacific J. Math. 123 (1986), 269-300.

[7] A. Garsia, Topics in Almost Everywhere Convergence, Markham, Chicago, 1970.

[8] M. S. Goldstein, Theorems of almost everywhere convergence in von Neumann algebras, J. Operator Theory 6 (981), 233-311 (in Russian).

[9] M. Goldstein and S. Litvinov, Banach principle in the space of $\tau$-measurable operators, Studia Math. 143 (2000), 33-41.

[10] G. Ya. Grabarnik and A. A. Katz, Ergodic type theorems for finite von Neumann algebras, Israel J. Math. 90 (1995), 403-422.

[11] R. Jajte, Strong Limit Theorems in Noncommutative Probability, Lecture Notes in Math. 1110, Springer, Berlin, 1985.

[12] A. Katok and B. Hasselblatt, Introduction to the Modern Ergodic Theory of Dynamical Systems, Cambridge Univ. Press, 1995.

[13] U. Krengel, Ergodic Theorems, de Gruyter, Berlin, 1985.

[14] S. Litvinov and F. Mukhamedov, On individual ergodic theorems in von Neumann algebras, Studia Math. 145 (2001), 55-63.

[15] E. Nelson, Notes on non-commutative integration, J. Funct. Anal. 15 (1974), 103116. 
[16] C. Ryll-Nardzewski, Topics in ergodic theory, in: Lecture Notes in Math. 472, Springer, 1975, 131-157.

[17] R. Sato, Operator averages for subsequences, Math. J. Okayama Univ. 22 (1980), 161-168.

[18] I. E. Segal, Non-commutative extension of abstract integration, Ann. of Math. 57 (1953), 401-457.

[19] E. Stein and G. Weiss, Introduction to Fourier Analysis on Euclidean Spaces, Princeton Univ. Press, 1971.

[20] M. Takesaki, Theory of Operator Algebras, I, Springer, New York, 1979.

[21] F. J. Yeadon, Non-commutative $L_{p}$-spaces, Math. Proc. Cambridge Philos. Soc. 77 (1975), 91-102.

[22] - Ergodic theorems for semi-finite von Neumann algebras, I, J. London Math. Soc. 16 (1977), 326-332.

IBM T.J. Watson Research Center

19 Skyline Dr.

Hawthorne, NY 10532, U.S.A.

E-mail: genady@us.ibm.com
Department of Mathematics Applied Mathematics and Astronomy University of South Africa Pretoria 0003, South Africa E-mail: lauralsh@gmail.com

Received February 8, 2006

Revised version March 28, 2007 